\title{
Data on the frequency of non-reproductive adults in a cross-cultural sample of small-scale human societies
}

\author{
Cody T. Ross - Paul L. Hooper - Monique Borgerhoff Mulder
}

\section{Background}

The goal of this brief communication is to report crosscultural data with relevance to researchers studying cooperative breeding in humans in the context of other non-human mammals. The data are derived from ethnographic research in 36 small-scale human populations/ cultural groups living on 5 continents.

A number of recent accounts have described Homo sapiens as a species that practices cooperative breeding [e.g., Hill and Hurtado (2009); Hrdy (2009); Kramer (2010); Mace and Sear (2005); van Schaik and Burkart (2010)]. These claims raise two important questions: first, do humans in general, or humans under a specific set of conditions, exhibit behaviors conforming to the technical definition of cooperative breeding? And second, to what extent are patterns of behavior and reproduction in humans similar to, or distinct from those found in non-human animals that are classified as cooperative breeders? These questions has been addressed in part through individual empirical cases studies (Hagen and Barrett, 2009; Hill and Hurtado, 2009; Kramer, 2005; Meehan et al, 2013; Strassmann, 2011) and theoretical papers (Burkart et al, 2009; Smaldino et al, 2013). They remain difficult to answer rigorously, however, without cross-cultural data (Hill et al, 2011; Kramer, 2010). While cooperative breeding is defined as adults exhibiting costly behaviors that typically increase the fitness of other adults, a likely consequence of such behavior is likely to be a pool of non-reproductive

Cody T. Ross

Santa Fe Institute, Santa Fe, NM 87501, United States

E-mail: ctross@ucdavis.edu

Paul L. Hooper

Department of Anthropology, Emory University, Atlanta, GA 30322, United States

E-mail: phooper@emory.edu adults. No studies to our knowledge have brought standardized cross-cultural data on the actual frequency of non-reproductive adults to bear on the topic. We provide such data in this brief communication.

We note that our data report not percentage of nonbreeders, but rather individuals with a measure of zero for various RS proxies (living children, children surviving to age 5, children surviving to age 21, etc.), and as such could reflect a number of different factors (such as: infertility, failure to keep children alive to 5th-or 21st - birthday, or reproduction suppression) that may, or may not, reflect cooperative breeding.

\section{Methods}

For purposes unrelated to questions concerning cooperative breeding in humans, we have compiled a large number of data sets on cross-cultural human reproductive outcomes, that may nevertheless be of interest to researchers studying cooperative breeding. Here, we outline our methods of: 1) data-set construction, and 2) Bayesian meta-analysis of the sex- and group-specific probably of remaining non-reproductive until a given age (we run the model for ages 25 and 45).

\subsection{Data}

Our data sets are wide ranging both spatially and temporally. For data to be included, we require that data collection be based on either total censuses or randomized samples of the population. For example, we do not consider data sets where only the reproductive outcomes of household heads are represented, as this could strongly bias our estimates of the frequency on nonreproductive adults. We detail the site-specific data collection methodology by field-site in Section 4 . 


\subsection{Bayesian Meta-Analysis}

In each population/cultural group, $j$, we count the total number of males and females with age $\geq 25$ in model 1 (and age $\geq 45$ in model 2 ), $N_{[j]}^{M}$ and $N_{[j]}^{F}$, respectively. We then count total number of males and females in this subset that have $R S=0, Z_{[j]}^{M}$ and $Z_{[j]}^{F}$. Following the multi-level meta-analysis model outlined in Ross et al (2015), we adopt a model where each population/cultural group has its own binomial probability distribution for remaining non-reproductive until age 25 in model 1 (and age 45 in model 2); we describe this probability distribution using the posterior distributions of parameters $\theta_{[j]}^{M}$ and $\theta_{[j]}^{F}$, which are unique to males and females respectively. These parameters are estimated using the population-specific count data and multi-level priors:

$Z_{[j]}^{M} \sim \operatorname{Binomial}\left(N_{[j]}^{M}, \theta_{[j]}^{M}\right)$

$Z_{[j]}^{F} \sim \operatorname{Binomial}\left(N_{[j]}^{F}, \theta_{[j]}^{F}\right)$

It should be noted that the posterior variances of $\theta_{R_{[j]}}$ and $\theta_{U_{[j]}}$, decrease as the sample size in a given population/cultural group increases, which automatically augments the weight that each population/cultural group carries on the estimation of higher order parameters. We utilize the inverse logit function to transform the probabilities, $\theta_{[j]}^{M}$ and $\theta_{[j]}^{F}$, into their respective log-odds expressions, $\Psi_{[j]}^{M}$ and $\Psi_{[j]}^{F}$, and declare that $\Psi_{[j]}^{F}$ can be described as $\Psi_{[j]}^{M}$ plus some deviation, $\delta_{[j]}$, which represents the estimated change in the log-odds of remaining non-reproductive as a function of sex, unique to each subpopulation, $j$.

$\Psi_{[j]}^{M}=\log \left(\theta_{[j]}^{M} /\left(1-\theta_{[j]}^{M}\right)\right)$

$\Psi_{[j]}^{F}=\log \left(\theta_{[j]}^{F} /\left(1-\theta_{[j]}^{F}\right)\right)=\Psi_{[j]}^{M}+\delta_{[j]}$

We then model the parameters $\Psi_{[j]}^{M}$ and $\delta_{[j]}$ as realizations from higher-level Gaussian distributions, with unknown means and standard deviations, to yield estimates corresponding to the mean log odds across populations of males remaining non-reproductive, and the mean offset in the log odds of males and females remaining non-reproductive:

$\Psi_{[j]}^{M} \sim \operatorname{Normal}\left(\mu_{\Psi}, \sigma_{\Psi}\right)$

$\delta_{[j]} \sim \operatorname{Normal}\left(\mu_{\delta}, \sigma_{\delta}\right)$
We utilize weakly informative normal priors on $\mu_{\Psi}$ and $\mu_{\delta}$, and weakly informative half-Cauchy priors on $\sigma_{\Psi}$ and $\sigma_{\delta}$ (Gelman et al, 2006):

$\mu_{\Psi} \sim \operatorname{Normal}(0,10)$

$\mu_{\delta} \sim \operatorname{Normal}(0,10)$

$\sigma_{\Psi} \sim \operatorname{Cauchy}(0,5) T[0, \infty]$

$\sigma_{\delta} \sim \operatorname{Cauchy}(0,5) T[0, \infty]$

We fit this model using Hamiltonian Monte Carlo, as implemented in the Stan 2.2.0 environment (Stan Development Team, 2013). Two chains were updated adaptively for 1,000 iterations, and then sampled for 3,000 iterations, with no thinning. Stan monitors multiple chain convergence with the $\hat{r}$ statistic (which equals 1 at convergence), and monitors effective sample size (Gelman and Rubin, 1992). All model parameters had an $\hat{r}$ statistic of less than 1.001, and an effective sample size of greater than 2,000. Visual inspection of trace plots showed excellent mixing and apparent convergence of multiple chains to the same equilibrium distribution. Model code and model diagnostics are included in the Supplementary Materials.

\section{Results}

Across study sites, the inferential results suggest that the median percent of non-reproductive adults older than 25 is 0.10 (PCI95: 0.06, 0.15) for males and 0.07 (PCI95: 0.03, 0.13) for females. The corresponding results for adults older than 45 are 0.05 (PCI95: 0.02, 0.09 ) for males and 0.04 (PCI95: 0.01, 0.09) for females. Figure 1 plots these distributions.

[Fig. 1 about here.]

There is a considerable range in the group- and sex-specific frequency of non-reproductive adults across sites, from near 0.00 (among men and women in a number of Tanzanian samples) to above 0.40 (among Meriam women and English men). Table 1 reports the following summary statistics for females and males at each study site: 1 ) the total number of adult age $\geq 25,2$ ) the total number and percent of adults age $\geq 25$ who did not produce any surviving offspring, and 3 ) the posterior estimates from the meta-analysis. Table 2 includes the same estimates but for adults age $\geq 45$. 
[Table 1 about here.]

[Table 2 about here.]

Finally, we investigate cross-cultural patterns in sexspecific odds of non-reproduction. For individuals of over 25 years of age, there is a general trend that women have lowered odds of being non-reproducers $\mu_{\delta}=-0.42$ (PCI95: -0.93, 0.06). For individuals over 45 years of age $\mu_{\delta}=-0.26$ (PCI95: $\left.-1.09,0.40\right)$, this estimate expands to straddle both sides of zero, indicating that across populations, there can be populations where etiher men or women have increased odds of being non-producers; as before, however, the bulk of this distribution lies on negative values, indicating a general trend that women generally have lowered odds of being non-reproducers. Figure 2 plots the density distributions of these posterior estimates.

[Fig. 2 about here.]

\section{Brief ethnographic information, and data collection methodology by site}

\subsubsection{Kipsigis (Kenya)}

The Kipsigis are farming and cattle herding population living in southwestern Kenya (Kericho, Rift Valley Province) (Borgerhoff Mulder, 1987a). They have a strong tradition of polygyny. Residence patterns are strongly virilocal, and the inheritance of land and livestock is strictly patrilineal (Borgerhoff Mulder, 1987b).

The data analyzed here were collected in 1981-1983, with some additional material from 1991. All households, in a cluster of different kokwetinwek (neighbourhoods), were visited and married individuals were interviewed; subsequently unmarried offspring for whom reproductive details were available from their parents were coded into the data set such that all reproductive aged individuals are now sampled. We consider individuals as non-reproductive if they failed to produce any offspring surviving to the age of five years. For younger individuals, age is typically known, but for older individuals a best estimate of age is generated based on ipinda (named circumcision age set) membership (Borgerhoff Mulder, 1987a). Full description of the data collection methodology can be found in (Borgerhoff Mulder, 1987a).

\subsubsection{Pimbwe (Tanzania)}

The Pimbwe population studied by Monique Borgerhoff Mulder are a horticultural population living on the north end of the Rukwa Valley in Mpanda District of Western Tanzania; they live off the production of subsistence crops (maize and cassava) and cash crops (maize, sunflower, and occasionally rice) (Borgerhoff Mulder, 2009a,b). They practice serial monogamy (both polyandry and polygyny), although a few men do maintain multiple wives concurrently, but rarely for more than a few years since divorce is common.

The data analyzed here were collected in several panels between 1995 and 2014, and represent a full census of the village of Mirumba (Borgerhoff Mulder, 2009a). To measure reproductive success ( $R S 5$, offspring born and survived to five years of age), we summed all reported children surviving to age five. See Borgerhoff Mulder (2009a) for further methodological details.

\subsubsection{Sukuma[2] (Tanzania)}

The Sukuma are a group of rapidly expanding agropastoralists in Tanzania (Paciotti and Borgerhoff Mulder, 2004; Paciotti et al, 2005). They are patrilocal and frequently polygynous (Paciotti and Borgerhoff Mulder, 2004). Their subsistence system is based on cattle husbandry and plow farming of rice, maize, peanuts, and potatoes (Paciotti and Borgerhoff Mulder, 2004).

The data analyzed here were opportunistically collected by Monique Borgerhoff Mulder in July-August of 2013. Respondents were typically adult male Sukuma who attended a ward-level seminar on HIV awareness. Follow up interviews were conducted at subsequent collective events, like weddings and village meetings, to get a sample that represents the majority of household heads over 40 years old in the Sukuma community of Kibaoni, Tanzanian and its sub-villages. Each man was interviewed privately. To measure reproductive success $R S_{5}$, we summed all reported children surviving to age five.

\subsubsection{Tyva (Southern Siberia)}

Tyva is a mountainous region in Southern Siberia, which was incorporated into the Soviet Union in 1914 and currently lies within the Russian Federation. The traditional economic base of the population of Tyva is semi-nomadic pastoralism based on sheep, goats, cattle, horses, yaks, camels, and reindeer.

The current sample was derived from retrospective demographic interviews collected in 2015 in two pastoral communities of the Bai-Taiga region of western Tyva. Interviews were conducted with all adults born prior to 1965 recording the reproductive histories of their parents, their siblings, and themselves. All individuals in the same generation as the interviewees born prior to 1945 were included in the analysis. 
Acknowledgements We thank Kim Hill, Joan Silk, and the members of the NIMBioS Working Group on Hierarchy and Leadership in Mammalian Societies for encouragement and helpful input. The data contributors thank their local assistants and the communities with whom they have worked for their cooperation and hospitality. NIMBIos has provide support for this project.

\section{References}

Borgerhoff Mulder M (1987a) On cultural and reproductive success: Kipsigis evidence. American Anthropologist $89(3): 617-634$

Borgerhoff Mulder M (1987b) Resources and reproductive success in women with an example from the Kipsigis of Kenya. Journal of Zoology 213(3):489-505

Borgerhoff Mulder M (2009a) Serial monogamy as polygyny or polyandry? Human Nature 20(2):130150

Borgerhoff Mulder M (2009b) Tradeoffs and sexual conflict over women's fertility preferences in mpimbwe. American Journal of Human Biology 21(4):478-487

Burkart JM, Hrdy SB, Van Schaik CP (2009) Cooperative breeding and human cognitive evolution. Evolutionary Anthropology 18(5):175-186

Gelman A, Rubin DB (1992) Inference from iterative simulation using multiple sequences. Statistical science pp 457-472

Gelman A, et al (2006) Prior distributions for variance parameters in hierarchical models. Bayesian analysis $1(3): 515-534$

Hagen EH, Barrett HC (2009) Cooperative breeding and adolescent siblings. Current Anthropology 50(5):727-737

Hill K, Hurtado AM (2009) Cooperative breeding in South American hunter-gatherers. Proceedings of the Royal Society B: Biological Sciences 276(1674):3863-3870

Hill KR, Walker RS, Božičević M, Eder J, Headland T, Hewlett B, Hurtado AM, Marlowe F, Wiessner P, Wood B (2011) Co-residence patterns in huntergatherer societies show unique human social structure. Science 331(6022):1286-1289

Hrdy SB (2009) Mothers and others. Belknap Press

Kramer KL (2005) Children's help and the pace of reproduction: cooperative breeding in humans. Evolutionary Anthropology: Issues, News, and Reviews $14(6): 224-237$

Kramer KL (2010) Cooperative breeding and its significance to the demographic success of humans. Annual Review of Anthropology 39:417-436

Mace R, Sear R (2005) Are humans cooperative breeders?
Meehan CL, Quinlan R, Malcom CD (2013) Cooperative breeding and maternal energy expenditure among aka foragers. American Journal of Human Biology 25(1):42-57

Paciotti B, Borgerhoff Mulder M (2004) Sungusungu: The role of preexisting and evolving social institutions among tanzanian vigilante organizations. $\mathrm{Hu}-$ man Organization 63(1):112-124

Paciotti B, Hadley C, Holmes C, Borgerhoff Mulder M (2005) Grass-roots justice in tanzania cultural evolution and game theory help to explain how a history of cooperation influences the success of social organizations. American Scientist 93:58-65

Ross C, Campiño P, Winterhalder B (2015) Frequencydependent social transmission and the interethnic transfer of female genital modification in the african diaspora and indigenous populations of colombia. Human Nature 26(4):351-377, DOI 10. 1007/s12110-015-9234-7, URL http://dx.doi.org/ $10.1007 / \mathrm{s} 12110-015-9234-7$

van Schaik CP, Burkart JM (2010) Mind the gap: cooperative breeding and the evolution of our unique features. In: Mind the Gap, Springer, pp 477-496

Smaldino PE, Newson L, Schank JC, Richerson PJ (2013) Simulating the evolution of the human family: Cooperative breeding increases in harsh environments

Stan Development Team (2013) Stan: A c++ library for probability and sampling, version 2.0. URL http: //mc-stan.org/

Strassmann BI (2011) Cooperation and competition in a cliff-dwelling people. Proceedings of the National Academy of Sciences 108(Supplement 2):10,89410,901 


\section{List of Figures}

1 Cross-cultural probability of being non-reproductive at age 25 (Frame 1a) and at age 45 (Frame 1b),

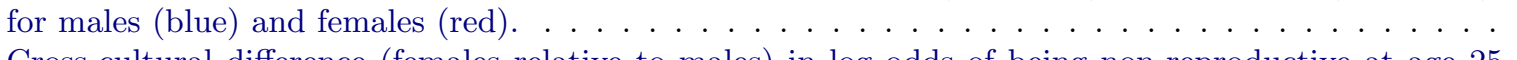

2 Cross-cultural difference (females relative to males) in log odds of being non-reproductive at age 25

3 Posterior estimates of the population-specific difference in $\log$ odds (females relative to males) of being non-reproductive at age 25 (Frame 3a) and at age 45 (Frame 3b). Thin lines denote the 90 percent posterior credibility intervals and the think lines denote the 50 percent posterior credibility intervals. Red points show the difference in log odds. Any bars without red points are posterior model predictions, but are based on missing data imputation. We note fairly extensive heterogeneity across population, with most populations showing reduced chances of females being non-reproductive relative to males at age 25 , but more equitable rates on non-reproduction at age 45 . 

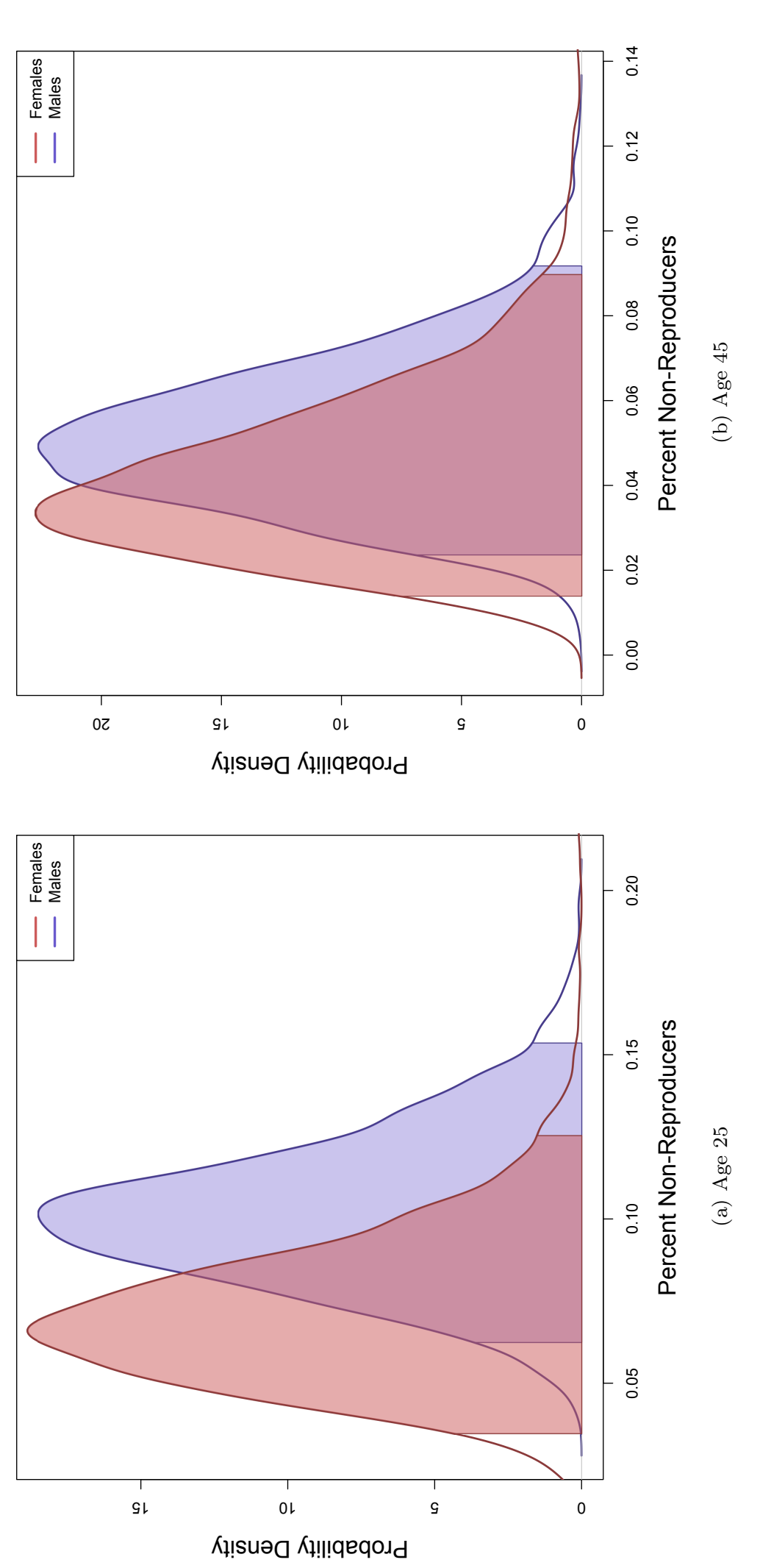

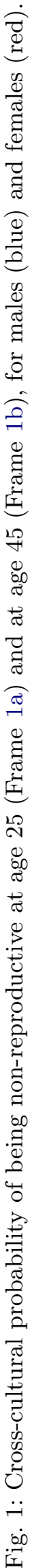




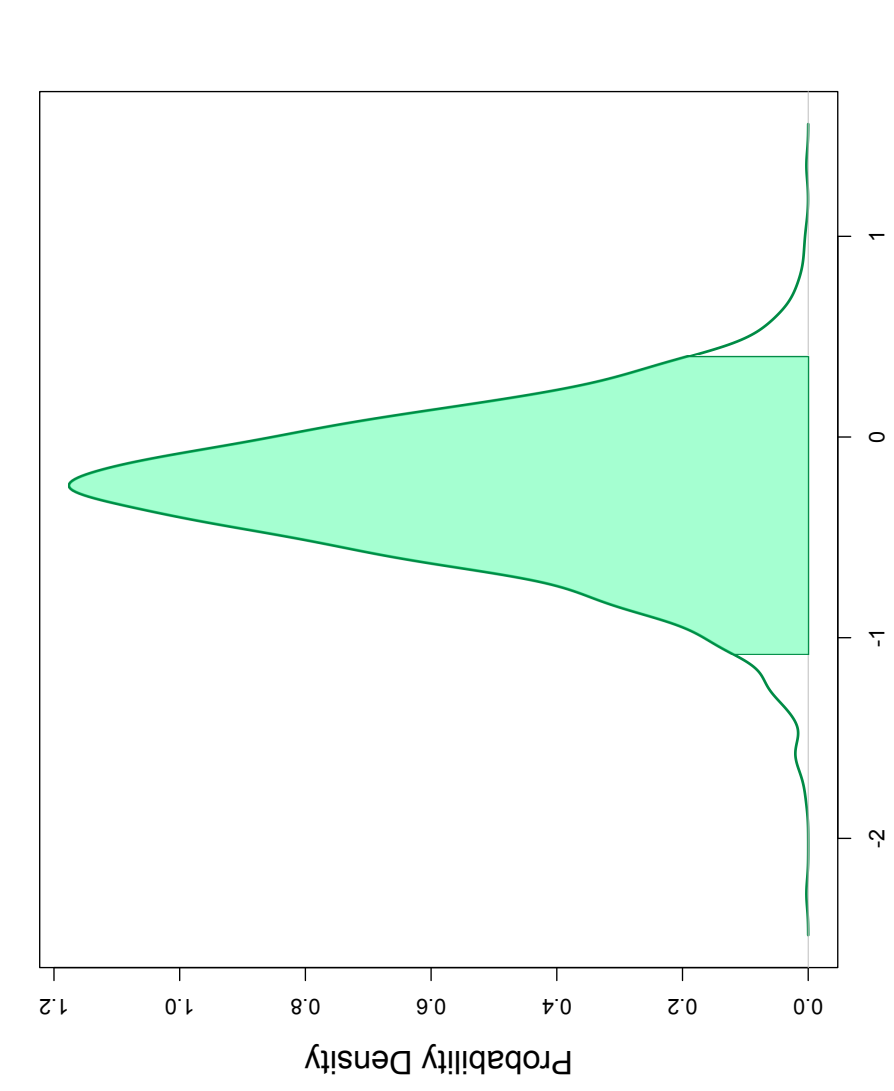

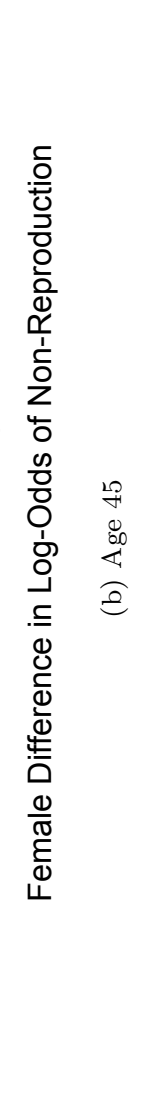




\section{List of Tables}

1 Frequency of non-reproductive adults age $\geq 25$. The symbols $N_{25}^{F}$ and $N_{25}^{M}$ give the sample sizes of males and females over the age of 25 in a given population, respectively. The symbols $Z_{25}^{F}$ and $Z_{25}^{M}$ give the corresponding sample sizes of males and females over the age of 25 who have $\mathrm{RS}=0$. The terms $F_{P c t}$ and $M_{P c t}$ give the raw percentages, and the terms $F_{P o s t}$ and $M_{\text {Post }}$ give the posterior results from the meta-analysis in terms of a median and a 95 percent posterior confidence region.

2 Frequency of non-reproductive adults age $\geq 45$. The symbols $N_{45}^{F}$ and $N_{45}^{M}$ give the sample sizes of males and females over the age of 45 in a given population, respectively. The symbols $Z_{45}^{F}$ and $Z_{45}^{M}$ give the corresponding sample sizes of males and females over the age of 45 who have $\mathrm{RS}=0$. The terms $F_{P c t}$ and $M_{P c t}$ give the raw percentages, and the terms $F_{P o s t}$ and $M_{\text {Post }}$ give the posterior results from the meta-analysis in terms of a median and a 95 percent posterior confidence region. . . . . . . 


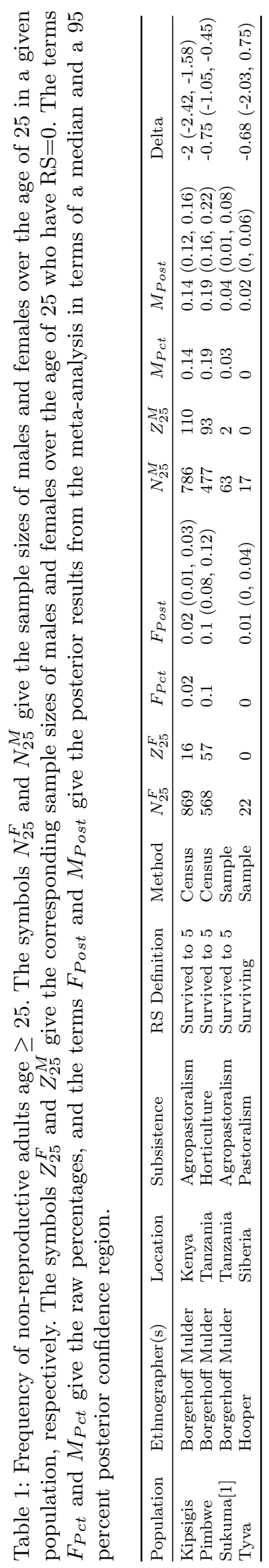




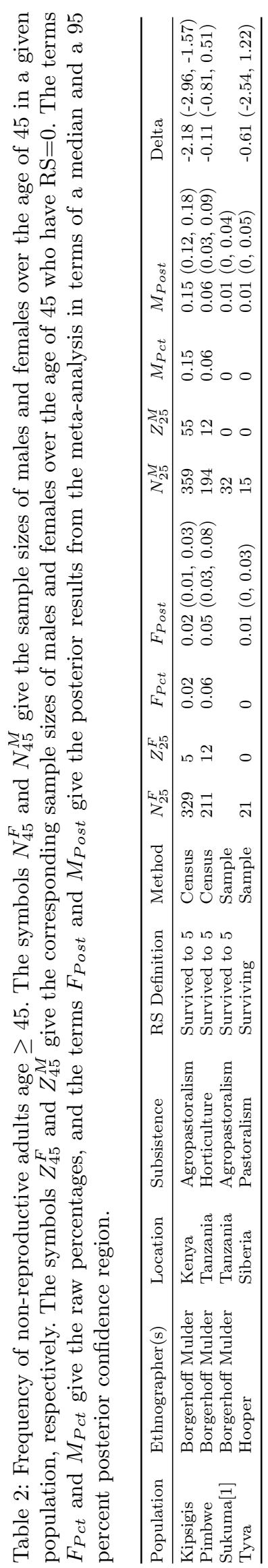

